\title{
Antropología
}

\section{El cuidado espiritual: una propuesta con base en las prácticas tradicionales de una comunidad indígena nahua}

\section{Spiritual Care: a traditional practices based proposal from nahua Indian Community}

\section{Cuidado espirituais: proposta com base nas práticas tradicionais de uma comunidade Nahua}

\author{
Eunice Victoria García Piña ${ }^{1}$, Marco Antonio Cardoso Gómez², Rosa María Ostiguín Meléndez ${ }^{3}$, \\ Virginia Reyes Audiffred ${ }^{4}$ \\ ${ }^{1}$ Maestra en enfermería. Universidad Nacional Autónoma de México \\ ${ }^{2}$ Doctor en antropología. Universidad Nacional Autónoma de México \\ ${ }^{3,4}$ Doctora en enfermería. Universidad Nacional Autónoma de México
}

Cómo citar este artículo en edición digital: García Piña, E.V., Cardoso Gómez, M.A., Ostiguín Meléndez, R.M., \& Reyes Audiffred, V. (2017). El cuidado espiritual: una propuesta con base en las prácticas tradicionales de una comunidad indígena nahua.

Cultura de los Cuidados (Edición digital), 21(48). Recuperado de < http://dx.doi.org/10.14198/cuid.2017.48.12>

Correspondencia: Eunice Victoria García Piña. Nahoas 150, Col. Pedregal de las Águilas. Del. Tlalpan. CP: 14439. México, D. F. Correo electrónico: eunice1388@hotmail.com Recibido: 12/01/2017; Aceptado: 28/03/2017

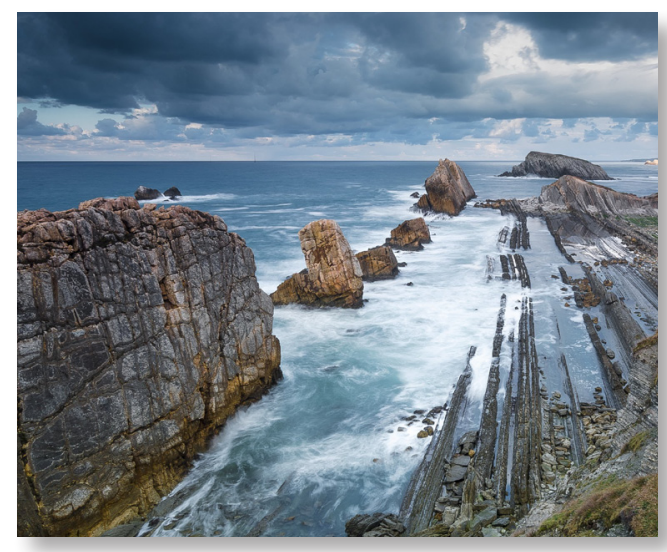

ABSTRACT

This paper outlines the findings from research named "Traditional and spiritual care practices in a Nahua, indigenous community" (García, 2015; García Piña, Cardoso Gómez, Serrano Sánchez \& Ostiguín Meléndez, 2015), in which spirituality elements were identified as coping strategies for the health-diseasedeath processes. Those results were complemented with a documental review.

Objective: to claim on the caring strategies, on a specific social, cultural and historical context, that promotes the improvement of the nursing practice in the Mexican context.

Method: theoretical-inductive documentary analysis. Results: communities are built from common goals and objectives, which arise as health-disease-death processes in its own particular perspective.

Conclusion: nursing has as one of its main functions, to rescue people's individual and collective response, for coping with every life situation; allowing that care itself, becomes a mutual feedback exercise.

Keywords: Nursing care, spiritual care, traditional practices, Indian comunnity. 


\section{RESUMEN}

El presente artículo se genera a partir de los resultados de la investigación "Prácticas de cuidado tradicional y espiritual en una comunidad indígena nahua” (García, 2015; García Piña, Cardoso Gómez, Serrano Sánchez \& Ostiguín Meléndez, 2015), donde se identificaron elementos de carácter espiritual como recurso para el afrontamiento del proceso salud-enfermedad y muerte. Dichos resultados se complementaron con un análisis documental.

Objetivo: Reivindicar las estrategias de cuidado en el contexto social, cultural e histórico, de la comunidad estudiada, con la finalidad contribuir a mejorar la práctica del cuidado de enfermería en el contexto mexicano.

Método: Análisis documental teórico-inductivo.

Resultados: Las comunidades se van construyendo a partir de objetivos y fines comunes, y son éstas mismas quienes generan respuestas a los procesos de salud-enfermedad y muerte desde su perspectiva particular.

Conclusiones: La enfermería tiene como función primordial rescatar las respuestas que las personas generan individual y colectivamente, para afrontar situaciones de la vida; y permitir que el cuidado sea un ejercicio de retroalimentación mutua.

Palabras clave: Cuidado de enfermería, cuidado espiritual, prácticas tradicionales, comunidad indígena.

\section{RESUMO}

Este artigo tem sua origem no projeto de pesquisa intitulado "Práticas de cuidado espiritual e tradicional em uma comunidade indígena Nahua” (García, 2015; García Piña, Cardoso Gómez, Serrano Sánchez \& Ostiguín Meléndez, 2015), no qual elementos de espiri- tualidade foram identificados como cópias de estratégias para o processo saúde-enfermidade-morte. Os resultados foram complementados com uma revisão documental.

Objetivo: reivindicar as estratégias assistenciais, em um contexto social, cultural e histórico específico, que promova a melhoria da prática de enfermagem no contexto mexicano.

Método: análise documental teórico-indutiva.

Resultados: as comunidades são construídas a partir de metas e objetivos comuns, que surgem como processos saúde-enfermidade-morte em sua própria perspectiva particular.

Conclusão: a enfermagem tem como uma de suas principais funções, resgatar a resposta individuais e coletivas das pessoas, com o objetivo de lidar com cada situação de vida; Permitindo, desse modo, que o cuidado torne-se uma mútua atividade de feedback.

Palabras-chave: cuidado de enfermagem, cuidado espirituais, prácticas tradicionais, comunidade indígena.

\section{INTRODUCCIÓN}

Antecedentes en la construcción sociocultural del cuidado.

La enfermería es la disciplina que se encarga del cuidado de las personas, con quienes establece una relación cercana en todas las etapas de la vida. En esta relación de cuidado se involucran aspectos humanos (físicos, emocionales, sociales, culturales, espirituales, entre otros) que constituyen parte fundamental en la relación enfermerapersona (García, 2015). Al hablar de cuidado a la persona, teóricamente se concibe a ésta en su totalidad, sin embargo, en la práctica diaria de la enfermería, poco se exploran las 
necesidades específicas del sujeto a quien se cuidará, y es entonces, cuando se otorga un cuidado alejado de sus expectativas. Como lo menciona Francisco del Rey (2008): "Los cuidados de salud de las personas y los grupos son la razón de ser de la enfermería, su objeto de estudio e interés. Esto que bien pudiera parecer una obviedad, no está tan claro ni a nivel general ni, lo que es más preocupante, entre las propias enfermeras".

En 1950, Madeleine Leininger, funda la llamada enfermería transcultural. "La autora identificó varias áreas comunes del saber y de intereses en la investigación teórica entre la enfermería y la antropología, formulando conceptos de enfermería transcultural, teoría, principios y prácticas" (Fawcett, 2013). Posteriormente, y hasta la actualidad, se integró una corriente disciplinar en algunos países, la denominada enfermería cultural o cuidados culturales que retoma los supuestos de Leininger para brindar cuidados acordes a las creencias y valores de las personas. En México, ha habido poca exploración de las necesidades de cuidado de sus poblaciones pese a ser un país con amplia diversidad cultural y poblacional. Por ejemplo, la medicina tradicional y complementaria (MTC) es una parte importante y con frecuencia subestimada de la atención a la salud (Organización Mundial de la Salud [OMS], 2013). Sin embargo, "las personas responden de forma distinta ante la enferme$\mathrm{dad}$, atendiendo a variables como puede ser la estructura social a la que pertenecen, las relaciones que establecen con las personas que interactúan y su medio, su sistema de valores y, por supuesto su cultura” (Cánovas, 2008); tal es el caso de las comunidades indígenas del país, quienes han establecido sus propias formas de cuidado acorde a su contexto histórico y cultural.
Con base en estos supuestos, se considera que existe la necesidad de acercarse más a la realidad social de los diferentes grupos sociales. El conocimiento de los aspectos ideológicos-culturales, brinda a la enfermería las herramientas para conceptualizar, planear e intervenir mejor. Tales aspectos son los que retoma la corriente de los cuidados culturales, basados en la "cultura, creencias de salud o enfermedad, valores y prácticas de las personas, para ayudarlas a mantener o recuperar su salud, hacer frente a sus discapacidades o a su muerte" (Leininger, 1999). Los beneficios que ofrecen los cuidados culturales se enfocan al sujeto que tiene una necesidad de ayuda en algún momento de la vida. Para tal efecto, el profesional de enfermería realiza un análisis integral del individuo, tomando en cuenta el padecimiento y los aspectos socioculturales y religiosos (Castillo, 2008). Este proceder, también beneficia a la disciplina enfermera, al generarle la oportunidad de brindar cuidados más contextualizados y por ende significativos.

La dimensión espiritual ha estado presente a lo largo de la historia de la enfermería, en teorías como la de Jean Watson quién expresa que la espiritualidad está relacionada con las respuestas humanas ante la salud y la enfermedad (Marriner, 2007).

El hecho de que teóricamente existan diferentes posturas para explicar el cuidado, ha conducido a la enfermería a reflexionar sobre la homogenización de los cuidados. Se ha llegado a la conclusión de que "no es posible homogeneizar el cuidado y pensar que los cuidados de una cultura, sean válidos para las demás" (Ramos, 2011). La literatura científica al respecto señala que existen diferentes necesidades de cuidado cultural y espiritual en las personas. 
Por ejemplo, en Colombia se realizó un estudio cualitativo que tuvo como finalidad crear modelos de cuidado en gestantes indígenas basados en las prácticas culturales de esa comunidad, al considerar relevante que el profesional de enfermería conozca y comprenda las creencias y costumbres de las comunidades indígenas (Ramos, 2011). También, se ha recurrido al uso de los modelos de enfermería para identificar aspectos culturales en una comunidad e introducir modelos profesionales de salud que contribuyan a su aceptación por parte de las personas (Pérez, 2009). Al concebir que cada cultura define sus tradiciones y costumbres visualizándolas desde su perspectiva de vida, porque en todas las etapas de la vida, las personas manifiestan necesidades de cuidado que estarán permeadas por las formas de ver la vida en los aspectos sociales, espirituales y culturales (Hernández \& Vásquez, 2010). Como en cada etapa de la vida, se construyen valores y creencias; la gestación, la maternidad y el nacimiento no están exentos de estas construcciones culturales (Laza, 2008), por lo cual, el cuidado de enfermería que se brinda debe ser coherente con su cultura (Hernández \& Vásquez, 2010). Todos los seres humanos necesitan ser cuidados en alguna etapa de la vida, por ello, la enfermera tendría que estar preparada y adaptarse a cada necesidad. De ahí que se considere como un desafío permanente en el cuidado de enfermería, identificar los valores, creencias y prácticas de los individuos que cuidan, además de manifestar respeto por la diferencia cuando ofrece dicho cuidado (Ibarra, 2006).

En la actualidad, el uso de la medicina tradicional responde a las necesidades de salud de diferentes grupos sociales, por lo que se convierte en un fenómeno insoslayable en las ciencias de la salud, a fin no sólo de reivindi- car reconocimiento sino de ser comprendida como una parte fundamental para el cuidado de la salud. Para muchos millones de personas, los tratamientos tradicionales representan la principal fuente de atención sanitaria, y a veces la única, porque esta forma de atención está próxima a los hogares, es accesible y asequible y además es culturalmente aceptada (OMS, 2013).

Los resultados del estudio "Prácticas de cuidado tradicional y espiritual en una comunidad indígena nahua" muestran que las prácticas de cuidado tienen su principal fundamentación en las prácticas de cuidado tradicional basadas en el uso de la herbolaria y masajes, así como los rituales de curación mediante la fe en un ser superior, referido como Dios, Madre Tierra o Universo. Lo anterior denota que la comunidad tiene la necesidad de buscar dentro de sus recursos, las pautas para un adecuado cuidado de su salud (García Piña et al, 2015).Es por condiciones como la anterior, que la OMS, propuso en el año 2013 una estrategia global para asegurar el acceso a la Medicina Tradicional Complementaria (MTC) y para promover la investigación, el desarrollo y la innovación con la finalidad de proteger los derechos de propiedad intelectual de los pueblos indígenas y las comunidades locales, así como de sus tradiciones de cuidados de salud.

La enfermería, como parte de un sistema de salud, también tendría que promover el cumplimiento de esta estrategia mediante el respeto y conocimiento de los tipos de cuidado a la salud y a la enfermedad que se generan en los diferentes grupos sociales y que permiten conservar la cultura, pero aún más importante, obedecen al mantenimiento del bienestar y la vida. 


\section{MÉTODO}

Se realizó una búsqueda documental en libros especializados y artículos científicos en bibliotecas e internet. Los artículos científicos se localizaron en bases de datos electrónicas a través de la combinación de las siguientes palabras clave: cuidado de enfermería, cuidado espiritual, cuidado cultural. Para el análisis, se utilizó el método de análisis documental teórico-inductivo que "orienta el manejo de las fuentes de información concretas, para leerlas, releerlas, analizarlas con amplitud y profundidad, y localizar y seleccionar los contenidos que servirán de fundamento a futuras concepciones y conclusiones sobre el tema que ya se está investigando" (Sánchez, 2000).

\section{RESULTADOS Y SU DISCUSIÓN}

Prácticas de cuidado en una comunidad indígena nahua.

En la comunidad indígena nahua llamada Tequila, ubicada en la Sierra Zongolica de Veracruz México, las prácticas de cuidado que se realizan, mismas que por su naturaleza, se clasifican en tradicionales y espirituales (García, 2015).

Las prácticas de cuidado tradicional, proporcionadas por un tepatih ${ }^{1}$, se enfocan a detectar los síntomas de los padecimientos y a abordarlos mediante un tratamiento culturalmente determinado, mientras que las prácticas de cuidado espiritual, brindadas por la ministra de la iglesia católica ${ }^{2}$, tienen como característica principal establecer un vínculo de acompañamiento en los procesos de enfermedad y muerte. Estas prácticas realizadas alternativamente generan un beneficio para la dualidad de cuidado, es decir, tanto para la persona que cuida como para la que es cuidada. Ambas prácticas se complementan, en tanto una trabaja la curación mediante la fe y el uso de recursos terapéuticos tradicionales (práctica tradicional), la otra se dedica a fortalecer la fe mediante la creencia en un ser superior (práctica espiritual), generando apoyo y cuidado en los procesos de salud enfermedad (García Piña et al, 2015). Los dos tipos de práctica se sustentan en la fe como elemento sustancial para la recuperación y/o mantenimiento de la salud.

\section{Beneficios del cuidado espiritual para la co- munidad de estudio.}

En Tequila se han construido, con base en los conocimientos empíricos, una serie de elementos sobre el cuidado espiritual que responden a necesidades culturales de cuidado, propias de una visión particular del mundo, que representan un significado para la comunidad, y han generado beneficios para sus habitantes. Se presentan tres, los que consideramos principales:

1. Afrontamiento individual y colectivo de los procesos de salud-enfermedad. La familia, la comunidad y la naturaleza forman parte de los elementos sustanciales para obtener el beneficio, y se observan a través del acompañamiento en la enfermedad y muerte, en el interés y dedicación por el otro mediante cuidados, tales como rezos, visitas para atender necesidades, tanto físicas como emocionales del enfermo, etc. Por ejemplo, la familia es participe de los rituales religiosos efectuados por la ministra de iglesia, quien cuida mediante cantos, rezos o presencia para sahumar ${ }^{3}$, además promueve el cuidado familiar mediante la integración grupal. De lo anterior deriva el segundo beneficio:

${ }^{1}$ Tepatih: referido en náhuatl como "el que cura".

${ }^{2}$ Ministra de iglesia católica: referente a la persona que se encarga de administrar los sacramentos de la iglesia.

${ }^{3}$ Sahumar: "Dar humo aromático a algo a fin de purificarlo o para que huela bien". (Diccionario de la Real Academia Española). 
2. Unión social, misma que les permite construir vínculos de ayuda mutua para la realización de diversas actividades y acompañarse en eventos importantes o difíciles de la vida. En consecuencia de estos beneficios, emerge el tercero:

3. Conservación de la vida, pues a lo largo de su historia, esta comunidad se ha mantenido y evolucionado con el uso de los recursos disponibles en su entorno inmediato, tales como las hierbas y su uso, así como el continuo recurrir a pedimentos y agradecimientos a la "Madre Tierra" o un ser superior mediante rituales.

Lo anterior preserva y promueve el respeto por: a) la Madre Tierra, b) un ser superior y c) las personas, lo cual propicia que se genere un significado y/o propósito a la vida que permite afrontar determinadas crisis inevitables en la vida de toda persona. Como lo menciona Sánchez (2004): "la relación del individuo con Dios [como cada cual lo defina] es central y es a partir de ella que se definen las experiencias de tener significado y propósito en la vida, esperanza, confianza y fe en alguien fuera de sí mismo".

La espiritualidad ha sido, y continúa siendo, un elemento de apoyo para el ser humano en todos los procesos de la vida y principalmente en situaciones de vulnerabilidad como la enfermedad y la muerte (Sánchez, 2004). Lo anterior se manifiesta claramente en la comunidad de Tequila, sin embargo, no es un elemento propio de ella, pues en general, las poblaciones indígenas de México tienen una forma particular de ver el mundo, lo cual se comparte de alguna manera con el resto de la población mexicana no indígena.Y una vez que se muestra que existen grupos sociales que realizan prácticas de cuidado espiritual, es conveniente adoptar una postura abierta $y$

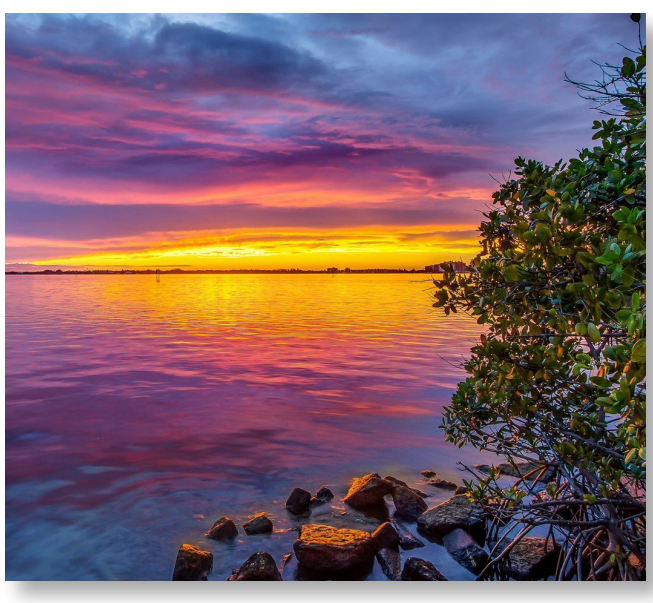

flexible al ejercer el cuidado, mirar desde la óptica de lo subjetivo y considerar el significado que aporta cada persona, para, con base en ello, convencerse que la labor de la enfermería no está sólo en función del tratamiento de la enfermedad desde una postura biológica, sino que involucra elementos de carácter social y cultural que pocas veces son abordados por la enfermería tanto a nivel comunitario como clínico. Una propuesta para ello es ofrecer cuidado al padeciente y su padecimiento (Cardoso, et al. 2014).

\section{Prácticas de cuidado en una comunidad in-} dígena y su relación con la enfermería.

En la comunidad de Tequila las prácticas de cuidado sientan sus bases en rituales de curación y religiosos donde la fe en un ser superior cumple un papel muy importante para el afrontamiento de los procesos de salud-enfermedad y muerte. Lo anterior, se ha considerado parte del cuidado espiritual al que ya en diferentes contextos han hecho alusión distintos autores (Navas \& Villegas, 2006).

De acuerdo a Navas \& Villegas (2006), la creencia en un ser superior y la fe podrían colocarse dentro del concepto espiritualidad, lo cual aporta un referente para retomar el cuidado espiritual como objeto de estudio de la 
enfermería en comunidades indígenas, pues es parte de su ideología social y cultural.

Con base en la trascendencia y beneficio otorgado a la comunidad, nuestra propuesta es retomar algunos elementos de dicho cuidado espiritual para dirigirlos a toda persona a la que se cuida, ya sea en comunidad indígena, rural o urbana. A continuación, se enlistan siete estrategias que, conforme a las necesidades de la persona, pueden retomarse para la práctica cotidiana de cuidar:

1. Escuchar las necesidades espirituales de las personas, implica otorgar un valor a las expresiones de la persona sobre su propio estado de salud, esto es, con los datos subjetivos como dolor, sensaciones, sentimientos, creencias, entre otros, y complementar con el diagnóstico objetivo de la enfermedad el cual se basa en datos como los signos vitales, exámenes de laboratorio, imagenológicos, y otros.

2. Tratar estos signos y síntomas inmersos en un elemento subjetivo de bienestar, mediante la fe depositada en el cuidado para promover la espiritualidad, a nivel individual y comunitario, en el afrontamiento de la enfermedad, de la muerte y para la recuperación de la salud.

3. Aceptar y respetar las creencias de las personas en la elección del tratamiento, ya sea tradicional, biomédico o cualquier otro, sin rehusar que la cultura determina las prácticas de la salud de las personas.

4. Planificar y brindar cuidado bajo la perspectiva de acompañamiento, sustentado en lo que el sujeto de cuidado expresa como sus necesidades, ya sean físicas, psicológicas o espirituales.

5. Planificar el cuidado del otro a partir de un vínculo subjetivo-afectivo, es decir, propiciar un acercamiento empático y compasivo con las personas al involucrar elementos afectivos respecto al dolor, miedo, concepción de la vida, sentido a la existencia, esperanza, entre otros aspectos que involucran sentidos diferentes en cada persona y que no son ajenos a las necesidades de cuidado en la saludenfermedad y muerte, con la finalidad de reconocer sus necesidades y planear un cuidado significativo.

6. Recuperar el concepto dual del cuidado de las comunidades indígenas, que se caracteriza por una relación persona-persona, donde impera la premisa: respeto y búsqueda del beneficio personal y comunitario a través de la ayuda mutua.

7. La fe en un Ser superior como elemento de bienestar subjetivo para afrontar la enfermedad, permite a la persona encontrar certidumbre sobre lo que sucederá después (curación, alivio, muerte). La persona creyente desarrolla esa capacidad sobre sí mismo, y tiene la posibilidad de encontrar un sentido a su vida mediante la esperanza y confianza en algo más, que le ayuda a transitar la enfermedad.

\section{CONCLUSIONES}

Resulta conveniente que se haga una reflexión hacia los modelos de cuidado utilizados, que están descontextualizados de la cultura mexicana, y comenzar a crear alternativas de cuidado que surjan de quien los recibe y no de quien los otorga. La forma de lograrlo puede ser a través de explorar en todo momento lo que el otro necesita y darle voz a sus necesidades (Cardoso, et al., 2007).

El cuidado, como objeto de estudio de la enfermería, requiere redefinirse a partir del reconocimiento de los aspectos subjetivos que son importantes para la persona; y dan significado al afrontamiento de los procesos de salud-enfermedad y muerte. Para tal efecto, la disciplina tendría que adaptar las ya existentes 
definiciones de cuidado a los diferentes grupos sociales o tener apertura a nuevas posibilidades de cuidado que se manifiesten en la práctica diaria.

Lo anterior, generara una clara visión y aceptación de que las comunidades se van construyendo a partir de objetivos y fines comunes, y son estas mismas quienes generan respuestas a los procesos de salud-enfermedad y muerte desde su perspectiva particular.

En este universo integrado por individuosociedad, inmerso en el proceso histórico, es donde la temporalidad del individuo desaparece para convertirse en la permanencia de la sociedad, y donde es posible explicar no sólo el comportamiento del individuo, sino el colectivo a través de la sabiduría, de la experiencia expresada en normas de ética, códigos de conducta social insertados en un patrimonio cultural propio (Barona \& Álvarez, 1986).

Lo anterior invita a reflexionar sobre ¿cuál debiera ser la labor de cuidado, tanto en comunidades indígenas como rurales o urbanas? La respuesta es evidente: conocer, acompañar, generando bienestar en la salud, la enfermedad y la muerte acorde al contexto socio-cultural de cada comunidad, con base en la subjetividad de la persona en su condición de sujeto y objeto de cuidado.

Pues como lo menciona Quilez \& Ferrer (2015): "el hombre tiene capacidad de conocerse e intervenir sobre sí, sobre los otros y sobre la naturaleza, es decir, es un ser responsable, capaz de responder".

Por tanto, la enfermería tendría como función primordial, actuar como acompañante y orientadora rescatando las respuestas que las personas generan individual y colectivamente, para afrontar situaciones de la vida, permitiendo que el cuidado sea un ejercicio de retroalimentación mutua. Para tal efecto, se propone considerar el cuidado espiritual como elemento de la enfermería, que implica el reconocimiento de una cosmovisión en donde el hombre existe en estrecha relación con la naturaleza, su comunidad, el otro y él mismo, para expresar subjetiva y objetivamente su experiencia de salud-enfermedad, y con base en ello, recibir una propuesta de cuidado a la salud pertinente en el marco del respeto a su libertad para decidir.

Mediante esta propuesta, se pretende preconizar el objetivo principal de la disciplina: cuidar la vida, mediante precisar un conjunto de actividades que generan beneficios terapéuticos para las personas de una comunidad indígena, y procurar extenderlos hacia comunidades rurales y urbanas.

\section{REFERENCIAS BIBLIOGRÁFICAS}

- Barona de Infante, N., \& Álvarez, L. (1986). El proceso de salud-enfermedad: un fenómeno social. Investigación y Educación en Enfermería, 4(2), 47-54.

- Cánovas Tomás, M. A. (2008). La relación de ayuda en enfermería. Una lectura antropológica sobre la competencia relacional en el ejercicio de la profesión. Tesis de doctorado. Murcia: Universidad de Murcia). Recuperado de http://bit.ly/2liy873

- Cardoso, M. A., Pascual, R., Moreno, G. M., Figueroa, M. E., \& Serrano, C. (2007). Investigación cualitativa y fenomenología en salud. Vertientes Revista Española en Ciencias de la salud, 10 (1-2), 25-32. Recuperado de http://bit.ly/2koaE6S

- Castillo, J. A. (2008). El cuidado cultural de enfermería. Necesidad y relevancia. Revista Habanera de Ciencias Médicas, 7(3), 1-7. Recuperado de: http://bit.ly/2ocmF8 - Fawcett, J., Desanto-Madeya, S. (2013). Contemporary Nursing Knowledge: Análisis and Evaluation of Nursing Models and Theories. Ed. F.A. Philadelphia: Davis Company.

- Francisco del Rey, J. C. (2008). De la práctica de la enfermería a la teoría enfermera. Concepciones presentes en el 
ejercicio profesional. Tesis de doctorado. Alcalá de Henares: Universidad de Alcalá de Henares. Recuperado de http://bit.ly/2ko1Bmr

- García Piña, E. V. (2015). Prácticas de cuidado tradicional y espiritual en una comunidad indígena nahua de Zongolica, Veracruz. Tesis inédita de maestría. México: Universidad Nacional Autónoma de México.

- García, P. E. V., Cardoso, G. M. A., Serrano, S. C., \& Ostiguín, M. R. M. (2015). Prácticas de cuidado tradicional y espiritual en una comunidad indígena nahua. Cultura de los cuidados, 19 (41), 34-43. doi: http://dx.doi. org/10.14198/cuid.2015.41.06

- Hernández, L., \& Vásquez, M. L. (2010). Practices and beliefs about exclusive breastfeeding by women living in Commune 5 in Cali, Colombia. Colombia Médica, 41 (2), 161-170. Recuperado de http://bit.ly/2166xJH

- Ibarra, T. X., \& Siles, J. (2006). Competencia cultural. Una forma humanizada de ofrecer cuidados de enfermería. Index de enfermería, 15 (55), 44-48. Recuperado de http://bit.ly/2lNjCYr

- Laza, C., \& Cárdenas, F. (2008). Una mirada al cuidado en la gestación desde la enfermería transcultural. Revista Cubana de Enfermería, 24(3), 1-7. Recuperado de http:// bit.ly/2knU6fn

- Leininger, M. (1999). Cuidar a los que son de culturas diferentes requiere el conocimiento y las aptitudes de la enfermería transcultural. Cultura de los cuidados, 3(6), 5-12. doi: http://dx.doi.org/10.14198/cuid.1999.6.01

- Moeini, M., Taleghani, F., Mehrabi, T., \& Musarezaie
A. (2014). Effect of a spiritual care program on levels of anxiety in patients whit leukemia. Iranian Journal of Nursing and Midwifery Research, 19(1), 88-93. Recuperado de http://bit.ly/2knXM0w

- Navas, C. \& Villegas, H. (2006). Espiritualidad y salud. Ciencias de la educación, 27 (1), 35-38. Recuperado de: http//bit.ly/2licXSu

- Estrategia de la OMS sobre medicina tradicional 20142023, Organización Mundial de la Salud, 2013. China. Recuperado de http://bit.ly/1Q3GLA5

- Pérez, S. (2009). Enfermería transcultural como método para la gestión del cuidado en una comunidad urbana. Revista Cubana de Enfermería, 25 (3-4), 1-8. Recuperado de http://bit.ly/lppnhXq

- Quilez Fajardo, L. y Ferrer Ferrándiz, E. (2015). Cuando la enfermería deviene teología. Cultura de los Cuidados, 19, 41, 23-33. doi: http://dx.doi.org/10.14198/ cuid.2015.41.05

- Ramos Lanfot, C. (2011). Prácticas culturales de cuidado de gestantes indígenas que viven en el Resguardo Zenú ubicado en la Sabana de Córdoba. Tesis de maestría- Bogota: Universidad Nacional de Colombia. Recuperado de http://bit.ly/2lNwWMv

- Sánchez Herrera, B. (2004). La dimensión espiritual del cuidado de enfermería en situaciones de cronicidad y muerte. Aquichán, 4, 6-9.Recuperado de: http://bit. ly/2INPnj1

- Sánchez, F. (2000). Prontuario de investigación documental y de campo. México: Trillas.

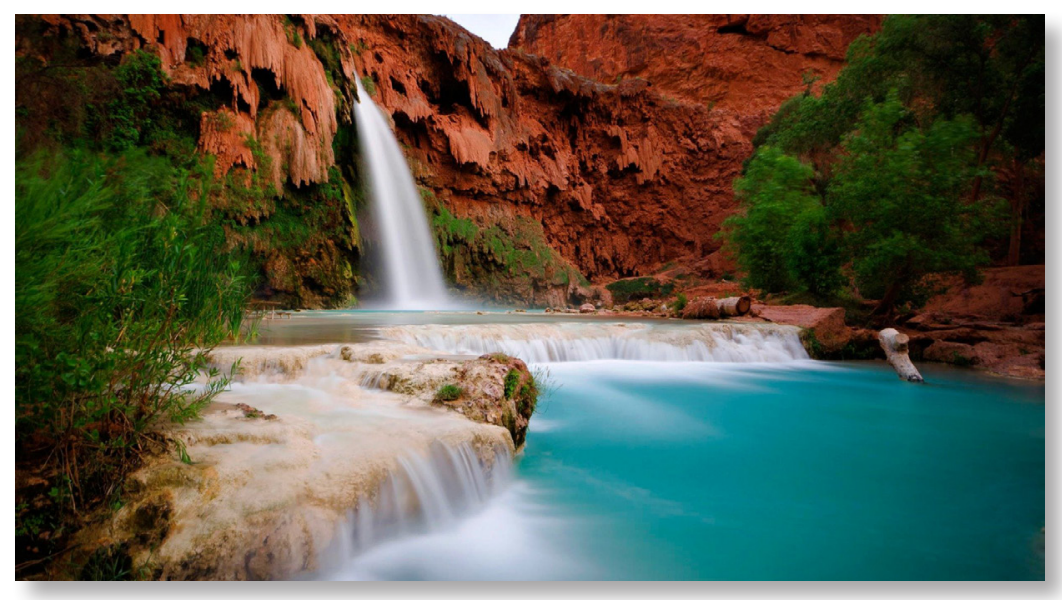

